\title{
Miniaturized Circularly Polarized Stacked Patch Antenna on Reactive Impedance Surface for Dual-Band ISM and WiMAX Applications
}

\author{
Kush Agarwal $^{1}$ and Saugata Dutta ${ }^{2}$ \\ ${ }^{1}$ Department of Electrical and Computer Engineering, National University of Singapore, 4 Engineering Drive 3, Singapore 117576 \\ ${ }^{2}$ School of Electrical and Electronic Engineering, Nanyang Technological University, 50 Nanyang Avenue, Singapore 639798 \\ Correspondence should be addressed to Kush Agarwal; agarwal.kush@nus.edu.sg
}

Received 30 August 2015; Revised 28 October 2015; Accepted 29 October 2015

Academic Editor: Maria E. De Cos

Copyright ( $) 2015$ K. Agarwal and S. Dutta. This is an open access article distributed under the Creative Commons Attribution License, which permits unrestricted use, distribution, and reproduction in any medium, provided the original work is properly cited.

\begin{abstract}
This paper proposes a compact microstrip patch antenna for operating in $2.4 \mathrm{GHz}$ ISM and $3.5 \mathrm{GHz}$ WiMAX bands with circularly polarized (CP) radiation. The CP radiation in dual-bands is a result of two multilayered truncated corner stacked square patches, while the reactive impedance surface (RIS) is used for antenna size miniaturization for the lower operating frequency band. Since the overall lateral antenna dimensions are controlled by the lower frequency band (higher wavelength), reducing the electrical size of the antenna for lower band results in overall smaller antenna dimensions. The measured 3-dB axial ratio bandwidths of the inhouse fabricated antenna prototype are $6.1 \%(2.40-2.55 \mathrm{GHz})$ for the lower band and $5.7 \%(3.40-3.60 \mathrm{GHz})$ for the upper band, while the $10-\mathrm{dB} S_{11}$ bandwidths for the two bands are $8.1 \%(2.39-2.59 \mathrm{GHz})$ and $6.9 \%(3.38-3.62 \mathrm{GHz})$, respectively. The maximum gain at boresight for the lower band is $2.93 \mathrm{dBic}$ at $2.5 \mathrm{GHz}$, while the gain for the upper band is $6.26 \mathrm{dBic}$ at $3.52 \mathrm{GHz}$. The overall volume of the proposed antenna is $0.292 \lambda_{0} \times 0.292 \lambda_{0} \times 0.044 \lambda_{0}$, where $\lambda_{\mathrm{o}}$ is the corresponding free-space wavelength at $2.5 \mathrm{GHz}$.
\end{abstract}

\section{Introduction}

Compact circularly polarized microstrip antennas (CPMAs) are useful for portable handheld devices because of their insensitivity to the change in polarization caused by the transmitting and receiving antenna orientations, as well as ionospheric rotation. For handheld and portable wireless communication devices, the antenna's overall volume is an important factor for the size of communication system. That is the reason why some commercial receivers use linearly polarized (LP) antennas to receive circularly polarized signals. LP antennas are smaller in size than the corresponding circularly polarized (CP) antenna [1], and 3-dB polarization mismatch loss is a trade-off for the reduction in antenna size. The problem is exacerbated when the antennas are supposed to radiate in two or more bands. There are many CPMAs proposed in the literature $[2,3]$. Generally, a single feed microstrip patch antenna generates a linearly polarized wave, unless some perturbation is introduced in the antenna patch to excite two orthogonal modes for $\mathrm{CP}$ radiation. This is usually achieved by making slots or slits $[4,5]$ or chamfering the patch radiator corners [6], the latter one being good for wide reflection coefficient and axial ratio bandwidth.

Till the beginning of this century, microstrip antennas were almost invariably fabricated on plane dielectric substrates. Since then, the introduction of artificial magnetic conductors (AMCs) has opened a new perspective to design smaller and more efficient microstrip antennas [7]. AMCs are also called high impedance surfaces (HISs), and from now these two terms will be used interchangeably in this paper. A modification of the AMC was suggested in [8]. These electromagnetic metasurfaces find application in enhancing the radiation properties of the antennas like operating frequency bandwidth, direction of antenna radiation, and size miniaturization. Both AMCs and RISs comprise periodic structures of different shapes and sizes [9]. The difference between the two is in their operating frequency. In a perfect electrical conductor (PEC), the image of an electric current 
parallel to the surface is always out of phase by $180^{\circ}$, irrespective of the frequency. But in a metasurface the phase difference between the current and its image (the reflection phase) varies from $180^{\circ}$ to $-180^{\circ}$, depending on the frequency of operation. At one particular frequency, the reflection phase is zero, and the image current is in the same phase as that of the physical current above it. At this frequency, the metasurface is AMC or HIS. When the reflection phase is $180^{\circ}$, the metasurface behaves like a normal PEC. At all other frequencies, the surface has reactive impedance and is called a reactive impedance surface.

Because of the difference in electrical properties, HISs and RISs have different applications when it comes to antenna design. HIS is more suitable for use as a reflector for antennas that have a bidirectional radiation pattern, where it limits antenna radiation in a particular direction. HISs are more effective than the conventional PEC reflectors because they can be brought really close to the radiating structure (separation as small as $0.1 \lambda_{\mathrm{o}}$ ) without distorting the antenna properties. Some monopole/dipole antennas have also been designed on HIS ground plane, thus resulting in low-profile antenna structures. On the other hand, RIS is a good substitute for the traditional high-dielectric substrate used for the size reduction. The inductive reactance of the RIS cancels the near-field capacitance of the microstrip antenna, resulting in a broader bandwidth and a compact size [8]. Besides these two general uses, there are metasurface based antennas that do not fall into these categories. Metasurfaces have been used to enhance the properties of both planar and nonplanar antennas. In $[10,11]$, a metasurface has been used to enhance $\mathrm{CP}$ radiation emitted from a dipole antenna and a patch antenna, respectively. Both these works involve a nonisotropic metasurface, and the reflection phases are $+90^{\circ}$ in the $x$-direction and $-90^{\circ}$ in the $y$-direction. That was achieved using periodic rectangular patches for designing the metasurface. Cross-dipole antennas over AMCs were studied in $[12,13]$. In both cases, the axial ratio performance of the intrinsically $\mathrm{CP}$ cross-dipole antenna is improved by the presence of the AMC surface. An on-chip CP antenna for use in the $60-\mathrm{GHz}$ Wi-Fi Band was presented in [14]. A CP antenna over a nonuniform HIS was presented in [15].

The main use of AMCs has been in making reflectors for bidirectional slot antennas. If designed properly, slot antennas radiate $\mathrm{CP}$ waves efficiently but are bidirectional in nature. To make them unidirectional, a reflector has to be used. A PEC reflector has to be kept at a minimum separation of $0.25 \lambda_{\mathrm{o}}$, or else the image current will cancel the main antenna current, resulting in no radiation [16]. This makes the antenna profile thick. The problem can be solved by using AMC reflector that can be brought very close to the radiating structure. The image current generated by the reflecting surface is now in phase with the antenna current for the optimized frequencies of operation. Hence the two do not cancel each other and the reflector can be brought very close to the main antenna, resulting in a low-profile antenna structure. This has been investigated in $[17,18]$ for different kind of slots and AMCs.

When RIS substrate is used in place of the tradition dielectric, it can increase the antenna bandwidth and reduce size. It does this by spatially distributing the image current so that there is minimum mutual coupling between the antenna current and its image. It also stores the magnetic energy that compensates for the near-field electric energy of the radiating structure [8]. It has been shown that the use of RIS substrate can result in the increase of both $S_{11}$ impedance bandwidth and the AR bandwidth for CP antennas. The use of RIS for reducing the patch size of single-layer microstrip antennas has been proposed in [19]. In [20], the metasurface structure has been modified by rotating the rectangular patches by $45^{\circ}$. This results in the RIS becoming even more optimized for $\mathrm{CP}$ radiation, with more AR bandwidth than a conventional RIS. The work of [21] describes a CP antenna loaded with composite right-handed/left-handed (CRLH) structures and optimized on RIS substrate. In [22], a single-patch single-band CP antenna with asymmetric slits and slots is proposed over RIS.

In this paper, $50 \mathrm{ohm}$ coaxial fed, stacked microstrip antenna over RIS is proposed and studied for CP radiation. The CP radiation in dual-bands $(2.4 \mathrm{GHz}$ ISM band and $3.5 \mathrm{GHz}$ WiMAX band) with compact size is achieved using two truncated corner square patch radiators stacked over RIS. The RIS is used in the inductive region for decreasing the resonance frequency for the lower band and improving the antenna radiation performance. The compact CP patch antenna loaded with RIS is designed, fabricated, and tested. Commercially available electromagnetic (EM) solver CST Microwave Studio (MWS) [23], based on the finite integration technique (FIT), is used for designing and optimizing the proposed antenna design. This paper is divided into four sections following this introduction. Section 2 explains in detail the concept of artificial impedance surface (AIS), AMC, and RIS. Section 3 describes the design of the metasurface and the patch radiators and how both interact with each other. The simulated and measured results are discussed in Section 4 . Section 5 concludes this paper by summarizing all the results obtained.

\section{AIS, AMC, and RIS}

The difference between RIS and AMC is their frequency of operation. To avoid confusion, the structure is called AIS in general. AIS can be modelled as a circuit comprising an inductance and capacitance in parallel. When the circuit is at the resonant frequency, it is AMC. At other frequencies, the net impedance is either inductive or capacitive and hence it is called RIS. The terms HIS and AMC can be used interchangeably. This is because two things can be observed at the resonance frequency. First of all, the resonant condition of a parallel LC circuit leads to a very high impedance (which is theoretically infinity) - hence the name HIS. And, secondly, the magnetic field tangential to the surface is zero (shown in Figure 1(b)). This is analogous to an electric conductor which has a zero tangential electric field (shown in Figure 1(a)). That is why these surfaces are called magnetic conductors. As they are not found in nature and are created artificially, the name given to them is artificial magnetic conductor (AMC).

The difference in properties between AMC and RIS is the result of difference in their reflection phase. In AMC, a charge and its image have the same phase. Hence a horizontal current 


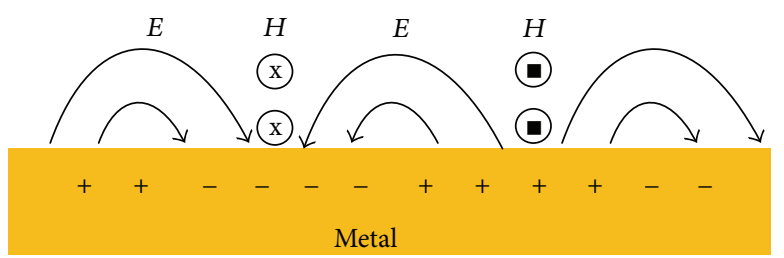

(a)

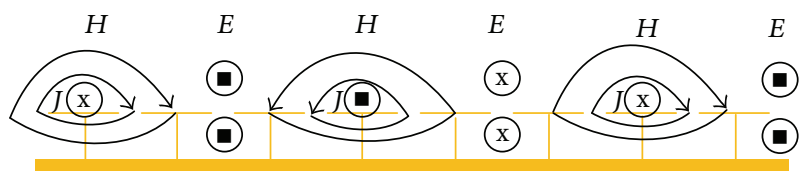

(b)

Figure 1: Comparison of electric field $E$ and magnetic field $H$ in (a) electric conductor and (b) magnetic conductor.
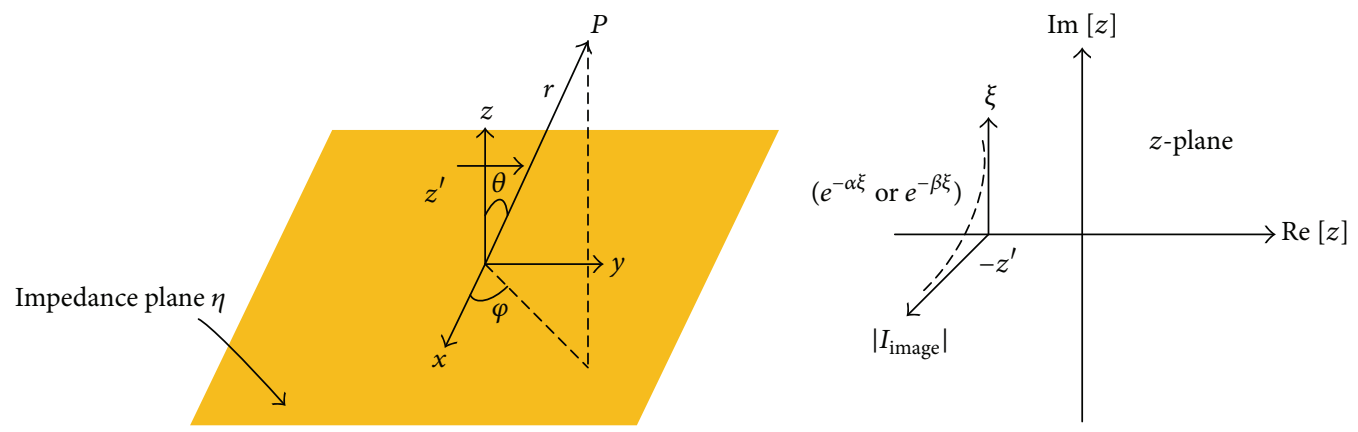

FIGURE 2: Image distribution of an infinitesimal dipole over a surface with impedance $\eta$ along the complex plane as in [8].

over AMC has the same orientation as its image current (it is opposite for a vertical current). This is in contrast with an electric conductor, which has the image out of phase from the main charge by $180^{\circ}$. For RIS working in the inductive mode, the reflection phase is between $180^{\circ}$ of an electric conductor and $0^{\circ}$ of a magnetic conductor. For a normal incident wave, the reflection phase of a surface with impedance $Z_{S}$ is given by

$$
\Phi=\operatorname{Im}\left[\ln \left(\frac{Z_{S}-\eta}{Z_{S}+\eta}\right)\right]
$$

where $\eta$ is the intrinsic impedance of free-space [24].

The effect of this is to push the image of an antenna current into a complex plane, so as to reduce the interaction (and consequently interference) between them as shown in Figure 2. The exact mathematical model is given in [8]. This causes the image current amplitude to vary with frequency so that at a certain frequency the image current has minimum amplitude as shown in Figure 3. Also, the normalized impedance can be chosen such that the stored energy in the image source compensates for the energy stored by the source itself; that is, if the antenna shows a capacitive loading and its image stores the magnetic energy, resonance can be achieved at much lower frequency than the resonance in free-space by proper integration with an inductive RIS.

The use of RIS substrate leads to an effective increase in the current path, thus leading to size reduction. Also, AIS is $\mathrm{AMC}$ at a single specific frequency, while it exhibits inductive behaviour over a wide range of frequencies.

\section{Proposed Antenna Geometry and Design}

The complete view and the cross-sectional view of the proposed antenna over RIS structure are shown in Figures 4(a) and 4(b), respectively. The antenna comprises two stacked truncated corner square patches (TCSP) placed on RIS substrate. The upper patch has a side length $L_{\text {up }}$ while the lower one has a side length $L_{\text {down }}$. The antenna is fed by $50 \Omega$ coaxial probe at a distance of $(x, y)$ from the patch centre. The RIS comprises an array of $5 \times 5$ periodic square metallic cell patches fabricated on a metal-backed FR4 substrate. This makes the antenna structure have three dielectric layers in total. The upper dielectric layer is made up of Rogers R04003 substrate $\left(\varepsilon_{r}=3.38\right.$ and $\left.\tan \delta=0.0027\right)$ and has a thickness of $h_{1}=1.6 \mathrm{~mm}$. The middle one is made up of FR4 substrate $\left(\varepsilon_{r}=4.2\right.$ and $\left.\tan \delta=0.02\right)$ with a thickness of $h_{2}=0.5 \mathrm{~mm}$, and the lowest layer is also of FR4 with a thickness $h_{3}=$ $3.2 \mathrm{~mm}$. The whole antenna is backed by a square-shaped metallic ground plane with side length of $L=35 \mathrm{~mm}$.

The RIS lies in the region between the two FR4 layers. In order to prevent shorting between the feed and the RIS patches, a circular region from RIS layer is removed to accommodate the coaxial SMA connector. The design of the RIS and the TCSP patch radiators is discussed in more detail in Sections 3.1 and 3.2, respectively.

3.1. Reactive Impedance Surface (RIS). The array of $5 \times 5$ square metal patch RIS under the patch radiators is shown in Figure 4(a). The first step to design RIS structure is to design its unit cell. The design parameter to optimize is the reflection phase, which is the phase of the reflected image when the surface is illuminated by TEM wave. The reflection phase of the unit cell is adjusted so that it lies between 

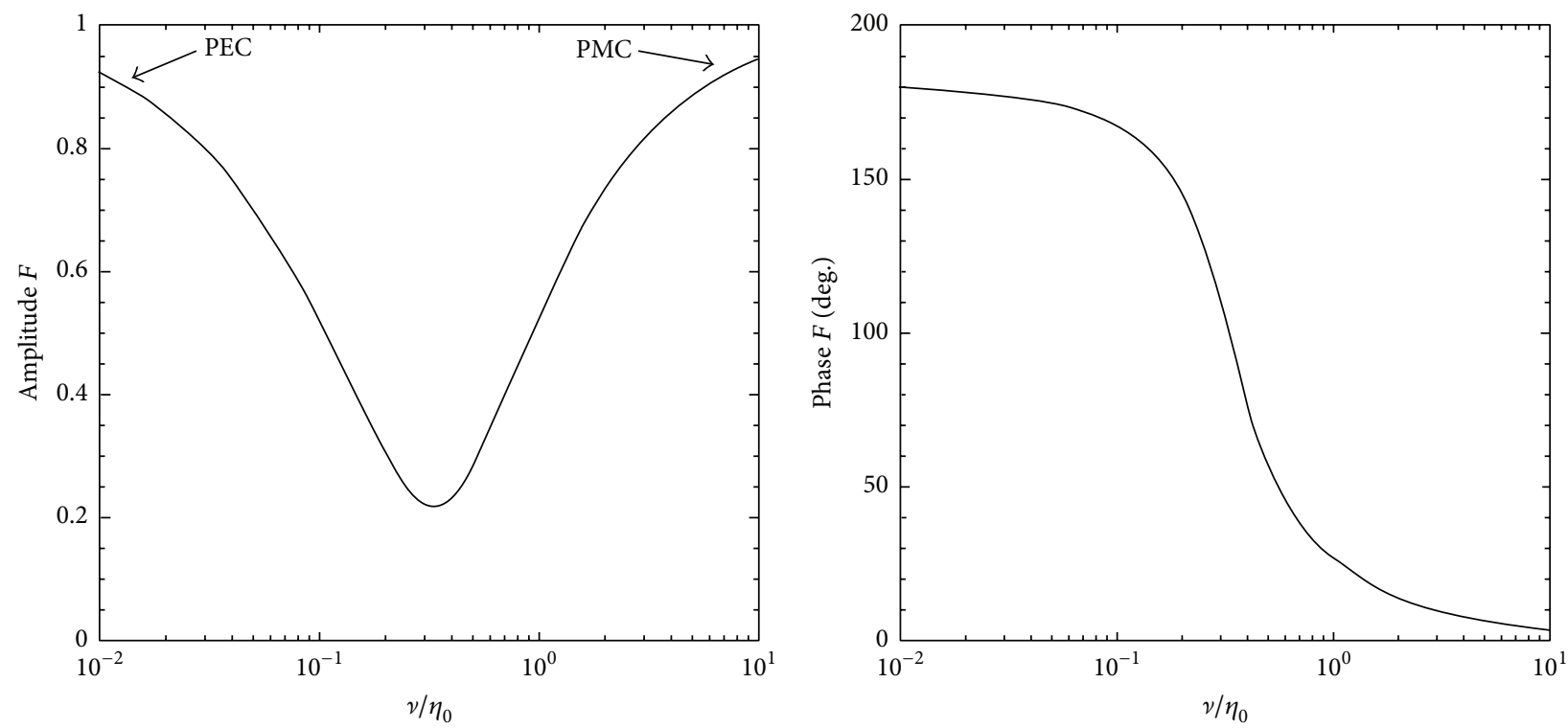

FIGURE 3: Variation of image amplitude and phase with frequency [8].

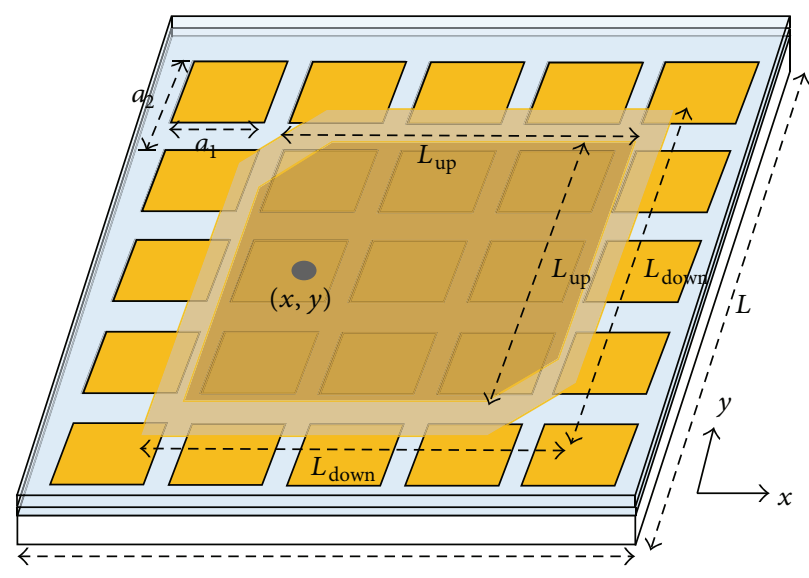

$L$

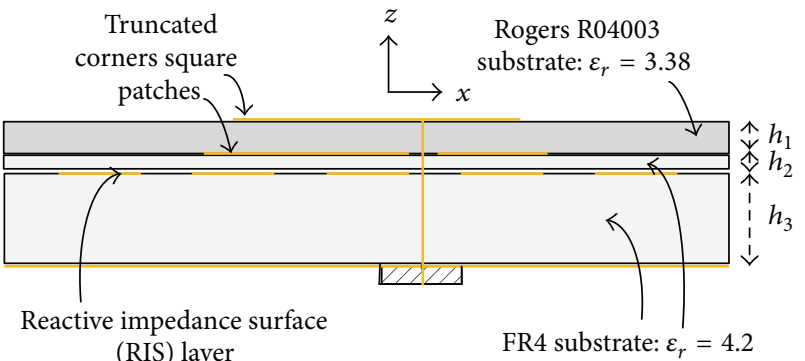

(b)

FIGURE 4: Proposed dual-band stacked microstrip patch antenna over RIS: (a) complete view of two TCSP stacked patches over $5 \times 5$ square patch RIS and (b) cross-sectional view of different stacked layers of FR4 and Rogers R04003 substrates.

the perfect electric conductor (PEC) $\left(180^{\circ}\right.$ reflection phase) and perfect magnetic conductor (PMC) $\left(0^{\circ}\right.$ reflection phase). While simulating the RIS, the patch parameter $a_{2}$ is kept fixed at $6.2 \mathrm{~mm}$, and $a_{1}$ is varied. The desired frequency of operation needs to be kept in the inductive impedance region. This gives the optimized value of $a_{1}$ as $5.4 \mathrm{~mm}$.

The use of RIS with purely reactive impedance has the following four advantages over the conventionally used PEC ground plane: (1) There is lower mutual coupling between the patch radiator and the ground plane, which allows for impedance matching over a wider bandwidth. (2) The inductive behaviour of the RIS compensates for the capacitive near field of the patch radiator, giving the antenna better radiation efficiency. (3) The inductive coupling reduces the antenna's resonant frequency, which effectively means that antennas at the same frequency will have a reduced size. (4) There is improvement in the front-to-back ratio [8].

3.2. Stacked TCSP Patch Radiators. The upper and lower TCSP radiators are shown in Figure 6. The CP radiation is generated using square patches that have the upper-left and lower-right corners chamfered by a certain length. This generates two orthogonal modes that are out of phase by $90^{\circ}$, resulting in CP radiation. The upper patch has a side length of $L_{\text {up }}=22.5 \mathrm{~mm}$ and is chamfered by a length of $l_{\text {up }}=3.25 \mathrm{~mm}$ in the two corners. The lower patch has a length of $L_{\text {down }}=24.0 \mathrm{~mm}$ and is chamfered by a length of $l_{\text {down }}=3.25 \mathrm{~mm}$. The coaxial feed is directly connected to the upper TCSP patch at a distance of $x=5.5$ and $y=0$ from the centre, while the lower TCSP patch is excited by EM 

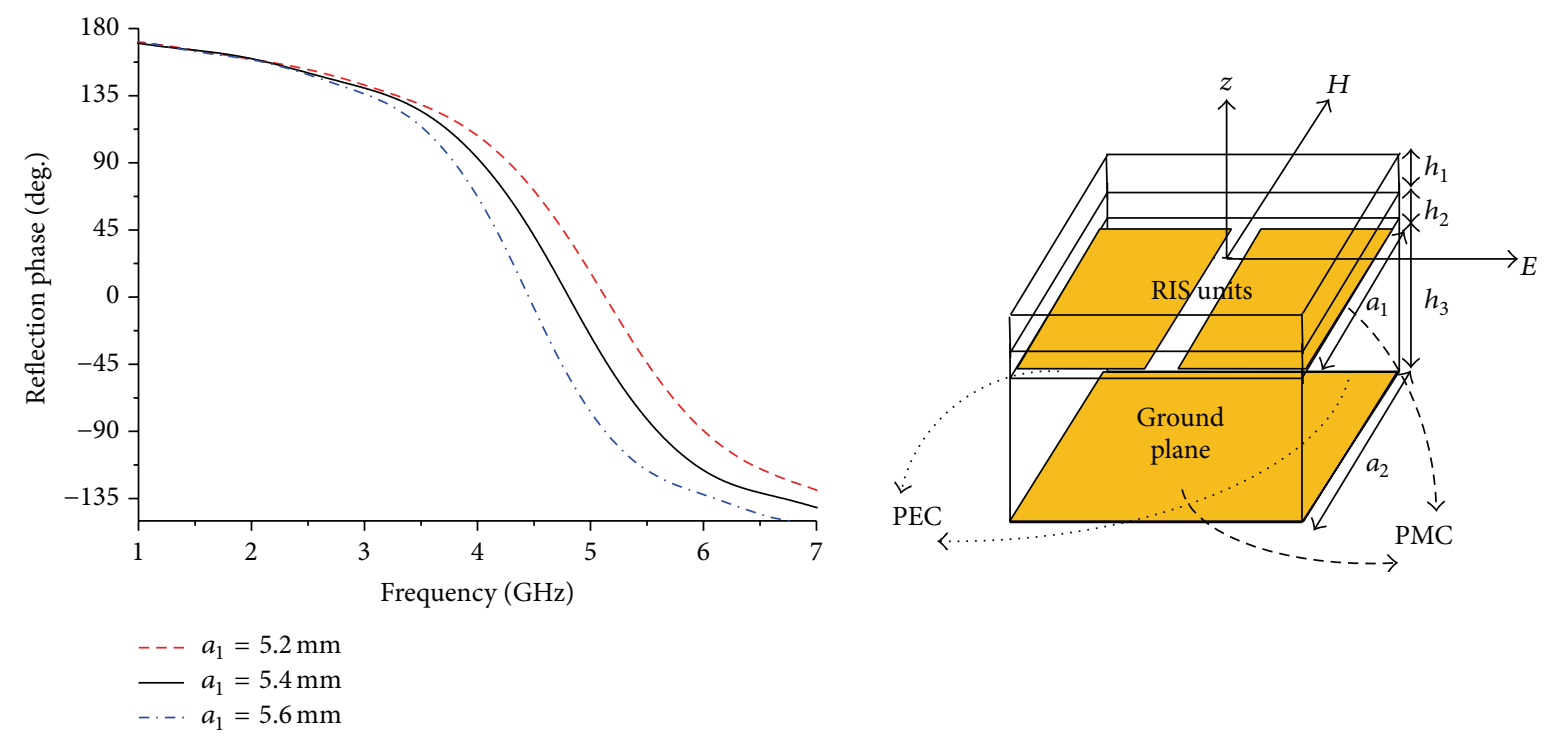

FIGURE 5: Reflection phase response with frequency for the RIS unit cell for different values of unit metal square patch dimension $\left(a_{1}\right)$ with the RIS unit cell enclosed by PEC walls in direction of $E$-field, PMC walls in the direction of $H$-field and illuminated by incident plane wave in negative $z$-direction.

TABle 1: Antenna dimensions (in $\mathrm{mm}$ ).

\begin{tabular}{lc}
\hline Parameter & Length \\
\hline$L_{\text {up }}$ & 22.5 \\
$l_{\text {up }}$ & 3.25 \\
$L_{\text {down }}$ & 24 \\
$l_{\text {down }}$ & 3.25 \\
$L$ & 35 \\
$h_{1}$ & 1.6 \\
$h_{2}$ & 0.5 \\
$h_{3}$ & 3.2 \\
$x$ & -5.5 \\
$y$ & 0 \\
$a_{1}$ & 5.4 \\
$a_{2}$ & 6.2 \\
\hline
\end{tabular}

coupling between the patches. All the antenna dimensions are summarized in Table 1 . The CP radiation for the upper band (3.5 GHz WiMAX band) is emitted by the upper patch of smaller dimensions, while the radiation of the lower band (2.4 GHz ISM band) comes from the lower patch of larger dimensions. Just like the case with the metallic RIS arrays, a circular region is removed from the lower TCSP radiator. This prevents it from getting shorted to the feed.

3.3. Performance of Antenna over RIS. The performance of $\mathrm{CP}$ antenna with this particular RIS metasurface has been studied in detail in [25]. Though this paper is focussed on a single-layer patch antenna, its results are directly applicable to the lower band of $2.4 \mathrm{GHz}$. The upper band is not very much affected by the RIS. This paper explored the effect of integrating the RIS with the antenna on the antenna's size, impedance bandwidth, and AR bandwidth. The reflection phase graph of a metasurface shows the reflection phase for a particular frequency. It depends on the shape and size of the metasurface, as well as the substrate and superstrate material permittivity and thickness. For the square patch metasurface of inner length $5.4 \mathrm{~mm}$ and outer length $6.2 \mathrm{~mm}$, the reflection phase graph is shown in Figure 5. The authors have designed an antenna for a set of frequencies corresponding to certain values of the reflection phase. In order to evaluate the performance of the metasurface, antennas have been made that are resonant at the same set of frequencies but do not contain a metasurface. In order to make the comparison accurate, the patch to ground plane separation is kept the same in both the cases.

It is observed that the presence of the RIS causes the reduction in patch size for the same operating frequency. The amount of reduction depends on the reflection phase the RIS is operating in, and it increases as the reflection phase approaches zero; that is, the patch size keeps getting smaller as the reflection phase approaches zero. However the reduction in patch size causes a reduction in the impedance bandwidth and gain. By the time reflection phase gets closer to $0^{\circ}$, the metasurface becomes more of HIS than RIS. In HIS, the image current has the same phase as the antenna current. Unlike RIS, the image current is not decoupled from the antenna current, and this reduces the antenna efficiency [8]. The usable bandwidth also becomes very limited. That is why the RIS is used at frequencies where the reflection phase is more than $+45^{\circ}$.

\section{Simulated and Measurement Results}

Keeping all the other parameters constant, the antenna was simulated with and without RIS. For an accurate comparison, the distance of the patch from the ground plane was kept the same in both the cases. The corresponding impedance 


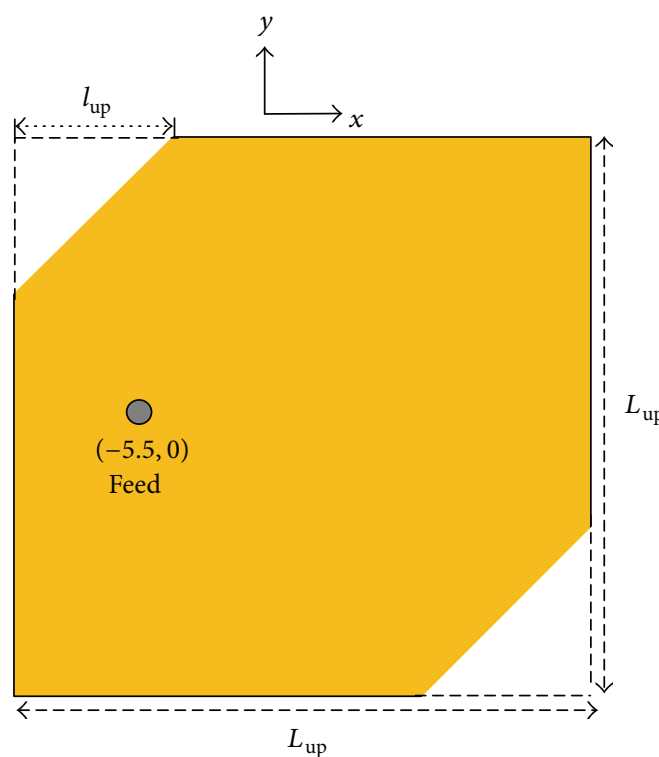

(a)

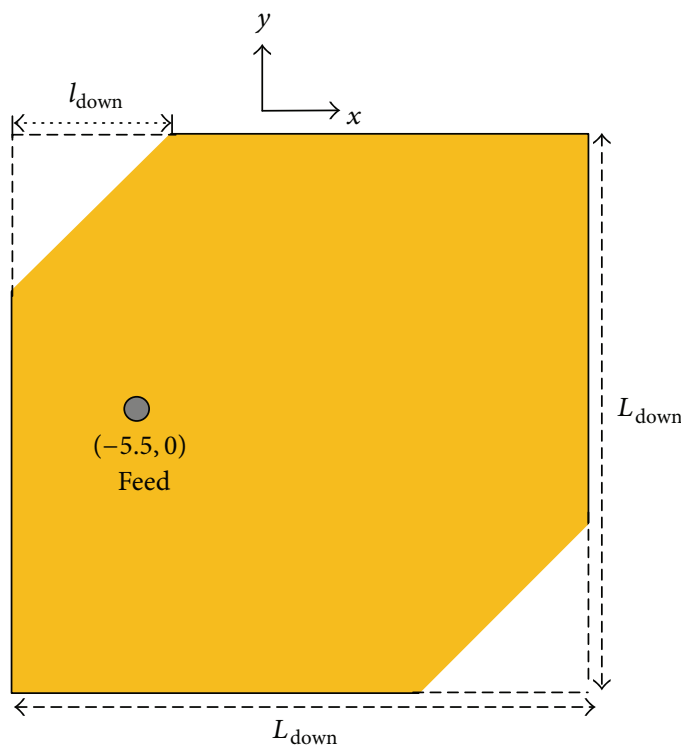

(b)

FIGURE 6: Top view of the proposed TCSP radiators over RIS: (a) upper and (b) lower patch.

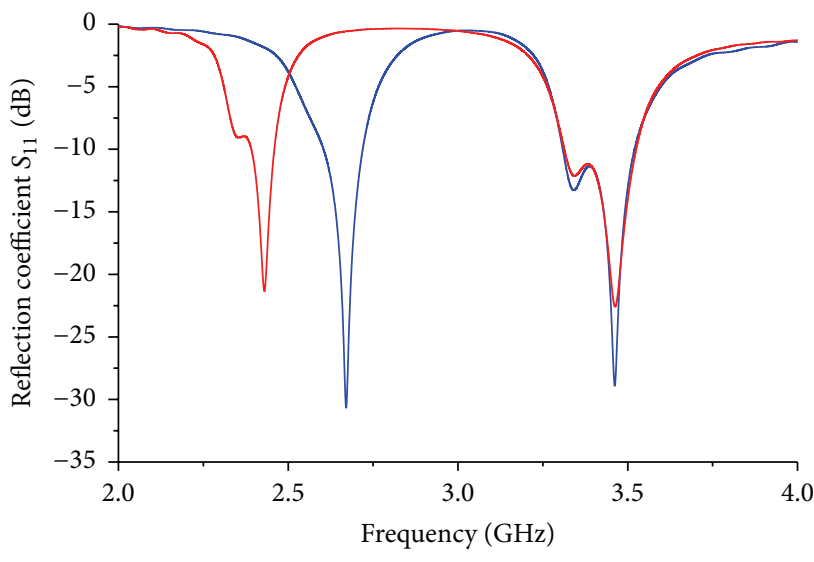

Without RIS
With RIS

(a)

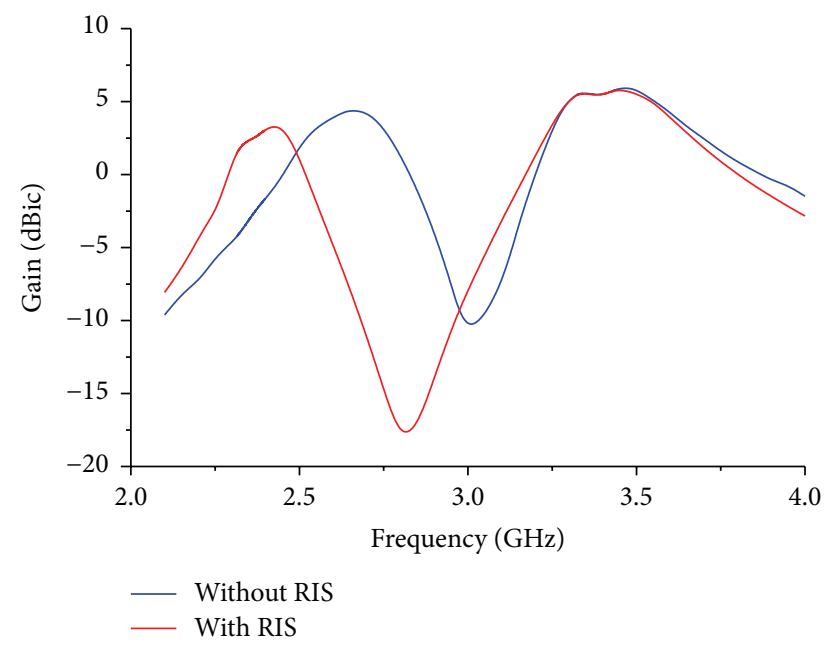

(b)

FIGURE 7: The variation of (a) 10-dB $S_{11}$ bandwidth and (b) gain versus frequency for the antenna structure with and without RIS.

bandwidth and gain plots are shown in Figures $7(a)$ and $7(\mathrm{~b})$, respectively. It can be observed that the presence of the RIS directly affects the lower band, because the lower frequency is radiated from the lower patch that is kept directly over the RIS. The RIS causes the resonant frequency of the lower band to decrease, because of the coupling of the RIS's inductive field with the patch radiator's near-field capacitance. As the same length of patch now radiates at a lower operating frequency, it makes the electrical length of the patch smaller (because of the wavelength radiated being now larger). This also reduces the antenna's gain slightly in the lower band, as that is a direct function of overall radiator size. The upper band is not affected by the RIS, as the patch radiator emitting that frequency is placed over a normal dielectric substrate. The use of RIS results in a $10 \%$ frequency reduction in the lower band. It also means that the radiation at the same frequency band can be obtained from a patch size smaller compared to the case without RIS. As the lower frequency band accounts for the overall lateral dimensions of the antenna, this corresponds to the overall size reduction of the antenna structure.

$\mathrm{CP}$ radiation is obtained from the patch antenna with its opposite corners chamfered. The perturbation introduced by the cut corners introduces an electric orthogonal field to the main field that is out of phase from it by $90^{\circ}$. This results in a rotating electric field and consequently $\mathrm{CP}$ radiation. The rotating electric fields can be visualized from Figures $8(\mathrm{a})$ and 8(b), which show the time-varying current distribution at the 

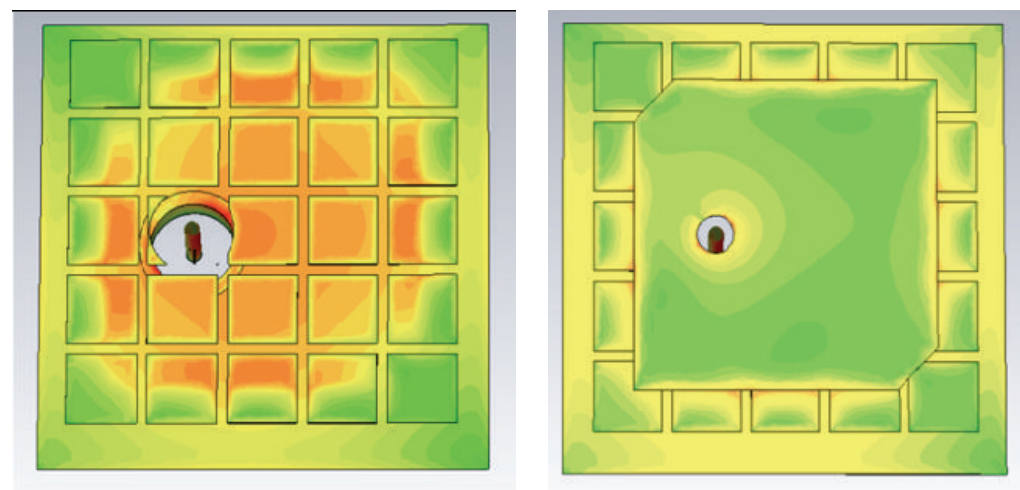

(a)
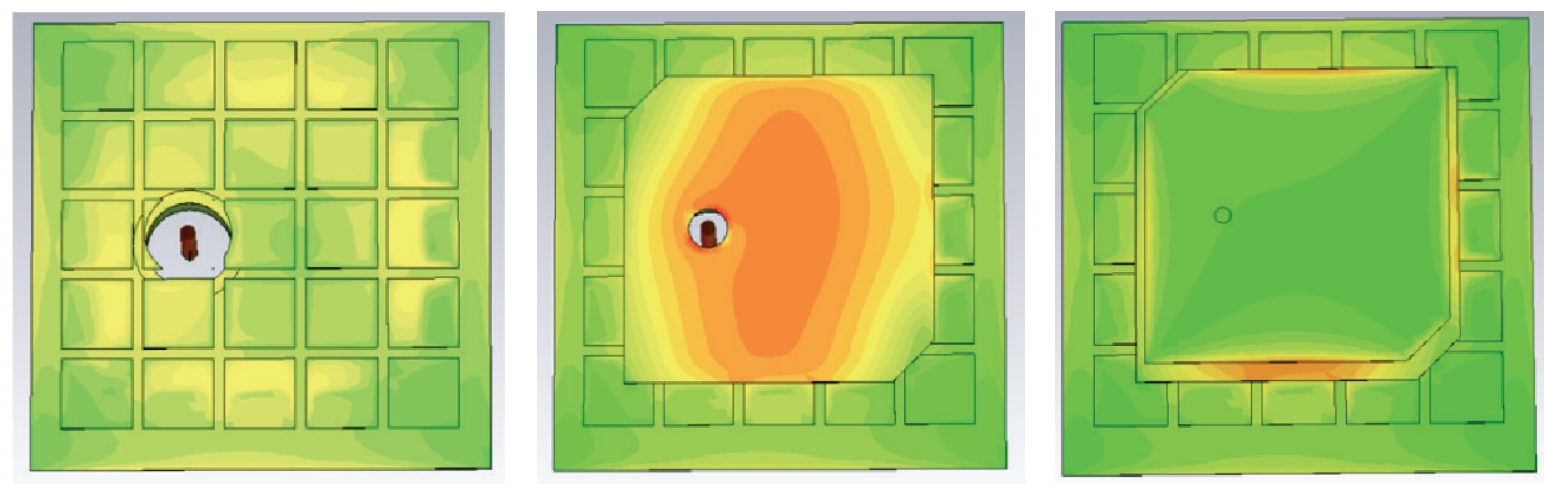

(b)

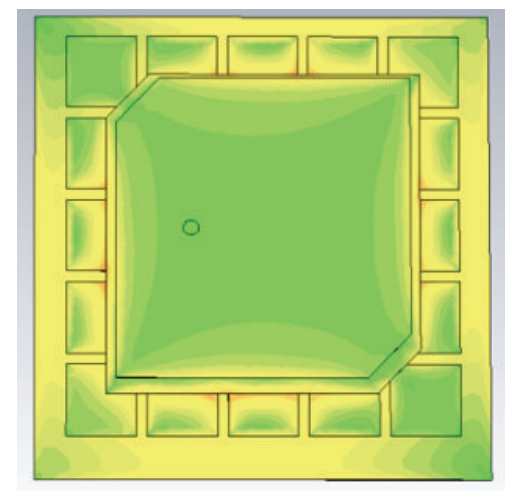



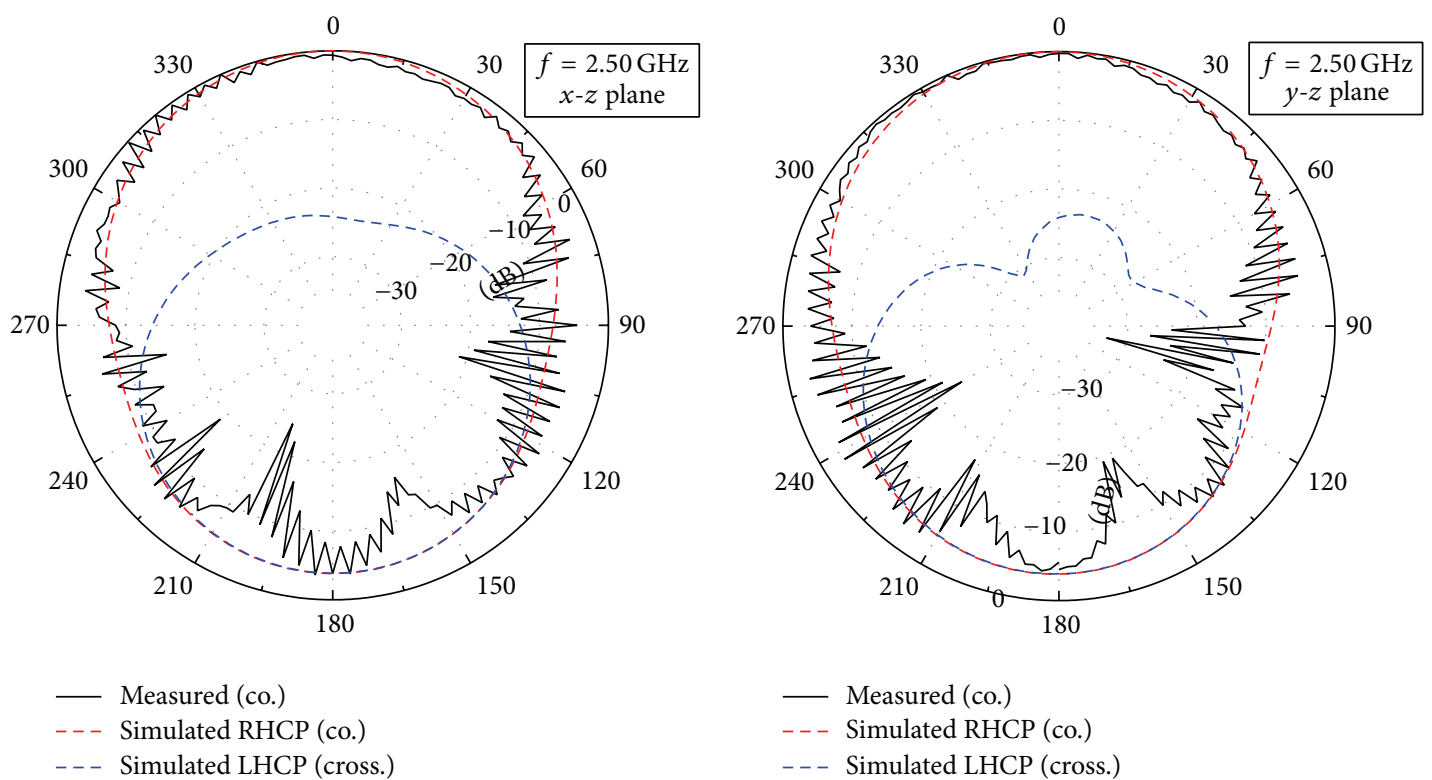

(a)

(b)

FIGURE 10: Measured and simulated (co- and cross-) normalized radiation patterns for both the principal planes at 2.50 GHz: (a) $x-z$ plane and (b) $y$ - $z$ plane.

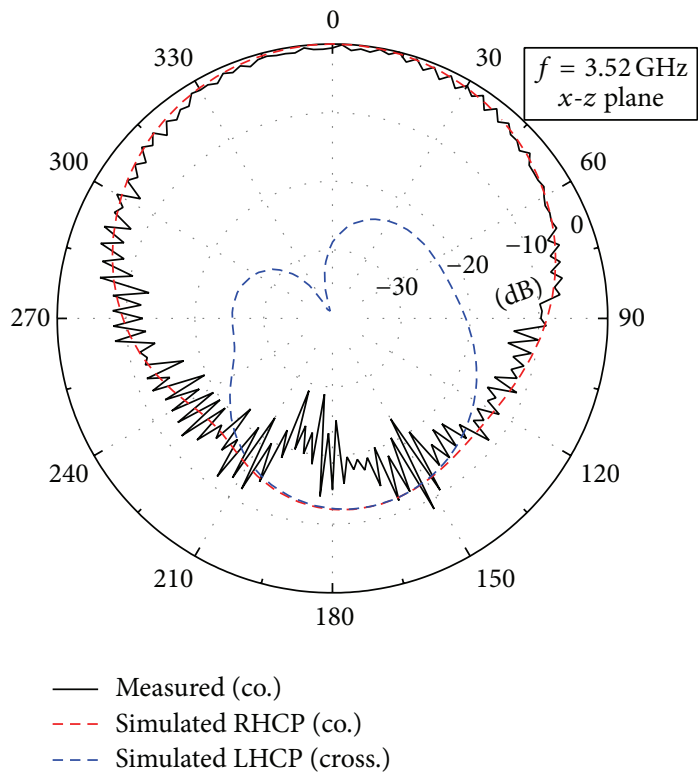

(a)

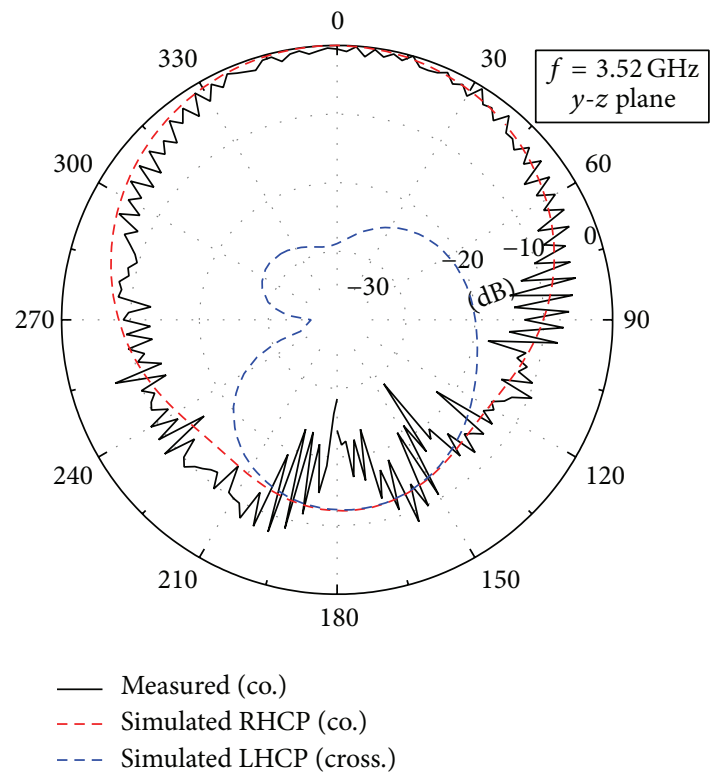

(b)

Figure 11: Measured and simulated (co- and cross-) normalized radiation patterns for both the principal planes at 3.52 GHz: (a) $x-z$ plane and (b) $y$ - $z$ plane.

(6.1\%) and 3.40 to $3.60 \mathrm{GHz}(5.7 \%)$ for the lower and upper bands, respectively. Global bandwidth is defined as the range of frequencies that are common to the impedance bandwidth and the AR bandwidth. The AR bandwidth lies completely within the impedance bandwidth, so the global bandwidth is the same as the AR bandwidth. The RHCP gain at the boresight is $2.93 \mathrm{dBic}$ at $2.50 \mathrm{GHz}$ and $6.26 \mathrm{dBic}$ at $3.52 \mathrm{GHz}$. The gain is almost constant over the global bandwidth, with a variation of $0.2 \mathrm{~dB}$.
The normalized radiation patterns for both the principal planes at the lower and upper frequency are shown in Figures 10 and 11, respectively. As the proposed antenna emits RHCP radiation, the RHCP pattern shows the copolarization level while the LHCP pattern indicates the cross-polarization level. At the lower frequency and at the boresight direction, the ratio of copolarization to cross-polarization level [RHCP LHCP] over the AR bandwidth is better than $24 \mathrm{~dB}$. At the upper frequency, the ratio of copolarization level is more 
than the cross-polarization level by $28 \mathrm{~dB}$. The $3-\mathrm{dB}$ CP beam width is more than $90^{\circ}$.

\section{Conclusion}

A compact, single feed, CP stacked microstrip antenna over RIS has been studied and presented for dual-band applications. The truncated corner method was used for both the square patches to generate $\mathrm{CP}$ radiation in the lower (2.4 GHz ISM) and upper $(3.5 \mathrm{GHz}$ WiMAX) bands. The design idea of reducing the overall antenna size was investigated by integrating the lower frequency (higher wavelength) radiating patch with RIS substrate. The size reduction takes place keeping other antenna parameters almost intact. If the patch size is kept constant, this technique results in radiation at a lower frequency. This method is useful as it leads to selective frequency reduction of a patch, while retaining the antenna properties and frequencies originating independently from other patches. It is especially useful for applications like GPS/GNSS as investigated in [26, 27], where relatively low frequency radiation (L-band) has to be generated from increasingly small sized antennas and the frequency ratio of multiple bands is less than 1 . This technique also has the advantage of easy integration with multilayer PCB technology, which makes the fabrication of low-cost and easy.

\section{Conflict of Interests}

The authors declare that there is no conflict of interests regarding the publication of this paper.

\section{References}

[1] X. Chen, C. G. Parini, B. Collins, Y. Yao, and M. U. Rehman, Antennas for Global Navigation Satellite Systems, John Wiley \& Sons, 2012.

[2] G. Kumar and K. Ray, Broadband Microstrip Antennas, Artech House, 2002.

[3] K.-L. Wong, Compact and Broadband Microstrip Antennas, vol. 168, John Wiley \& Sons, New York, NY, USA, 2002.

[4] Nasimuddin, Z. N. Chen, and X. Qing, "Asymmetric-circular shaped slotted microstrip antennas for circular polarization and RFID applications," IEEE Transactions on Antennas and Propagation, vol. 58, no. 12, pp. 3821-3828, 2010.

[5] X. Qing and Z. N. Chen, "Compact asymmetric-slit microstrip antennas for circular polarization," IEEE Transactions on Antennas and Propagation, vol. 59, no. 1, pp. 285-288, 2011.

[6] P. C. Sharma and K. C. Gupta, "Analysis and optimized design of single feed circularly polarized microstrip antennas," IEEE Transactions on Antennas and Propagation, vol. 31, no. 6, pp. 949-955, 1983.

[7] D. Sievenpiper, L. Zhang, R. F. Jimenez Broas, N. G. Alexöpolous, and E. Yablonovitch, "High-impedance electromagnetic surfaces with a forbidden frequency band," IEEE Transactions on Microwave Theory and Techniques, vol. 47, no. 11, pp. 2059-2074, 1999.

[8] H. Mosallaei and K. Sarabandi, "Antenna miniaturization and bandwidth enhancement using a reactive impedance substrate,"
IEEE Transactions on Antennas and Propagation, vol. 52, no. 9, pp. 2403-2414, 2004.

[9] A. Foroozesh and L. Shafai, "Investigation into the application of artificial magnetic conductors to bandwidth broadening, gain enhancement and beam shaping of low profile and conventional monopole antennas," IEEE Transactions on Antennas and Propagation, vol. 59, no. 1, pp. 4-20, 2011.

[10] F. Yang and Y. Rahmat-Samii, "A low profile single dipole antenna radiating circularly polarized waves," IEEE Transactions on Antennas and Propagation, vol. 53, no. 9, pp. 3083-3086, 2005.

[11] T. Nakamura and T. Fukusako, "Broadband design of circularly polarized microstrip patch antenna using artificial ground structure with rectangular unit cells," IEEE Transactions on Antennas and Propagation, vol. 59, no. 6, pp. 2103-2110, 2011.

[12] J.-M. Baracco, L. Salghetti-Drioli, and P. De Maagt, "AMC low profile wideband reference antenna for GPS and GALILEO systems," IEEE Transactions on Antennas and Propagation, vol. 56, no. 8, pp. 2540-2547, 2008.

[13] S. X. Ta and I. Park, "Circularly polarized dual-band crossed dipole antenna on an artificial magnetic conductor reflector," in Proceedings of the 7th International Congress on Advanced Electromagnetic Materials in Microwaves and Optics (METAMATERIALS '13), pp. 151-153, IEEE, Talence, France, September 2013.

[14] X.-Y. Bao, Y.-X. Guo, and Y.-Z. Xiong, "60-GHz AMC-based circularly polarized on-chip antenna using standard $0.18-\mu$ $\mathrm{m}$ CMOS technology," IEEE Transactions on Antennas and Propagation, vol. 60, no. 5, pp. 2234-2241, 2012.

[15] M. Hosseini and S. Bashir, "A novel circularly polarized antenna based on an artificial ground plane," Progress in Electromagnetics Research Letters, vol. 5, pp. 13-22, 2008.

[16] Y. Yoshimura, "A microstripline slot antenna (short papers)," IEEE Transactions on Microwave Theory and Techniques, vol. 20, no. 11, pp. 760-762, 1972.

[17] K. Agarwal, Nasimuddin, and A. Alphones, "Wideband circularly polarized AMC reflector backed aperture antenna," IEEE Transactions on Antennas and Propagation, vol. 61, no. 3, pp. 1456-1461, 2013.

[18] K. Agarwal, Nasimuddin, and A. Alphones, "Unidirectional wideband circularly polarised aperture antennas backed with artificial magnetic conductor reflectors," IET Microwaves, Antennas \& Propagation, vol. 7, no. 5, pp. 338-346, 2013.

[19] K. Agarwal, Nasimuddin, and A. Alphones, "RIS-based compact circularly polarized microstrip antennas," IEEE Transactions on Antennas and Propagation, vol. 61, no. 2, pp. 547-554, 2013.

[20] L. Bernard, G. Chertier, and R. Sauleau, "Wideband circularly polarized patch antennas on reactive impedance substrates," IEEE Antennas and Wireless Propagation Letters, vol. 10, pp. 1015-1018, 2011.

[21] Y. Dong, H. Toyao, and T. Itoh, "Compact circularly-polarized patch antenna loaded with metamaterial structures," IEEE Transactions on Antennas and Propagation, vol. 59, no. 11, pp. 4329-4333, 2011.

[22] K. Agarwal, Nasimuddin, and A. Alphones, "Compact asymmetric-slotted-slit patch based circularly-polarized antenna with reactive impedance surface substrate," Microwave and Optical Technology Letters, vol. 54, no. 11, pp. 2505-2510, 2012.

[23] CST, CST Microwave Suite Version 2012, CST, 2012, http://www .cst.com/. 
[24] D. F. Sievenpiper, "Artificial impedance surfaces for antennas," in Modern Antenna Handbook, pp. 737-777, 2008.

[25] K. Agarwal, N. Nasimuddin, and A. Alphones, "Design of compact circularly polarized microstrip antennas using metasurfaces," in Proceedings of the 43rd European Microwave Conference (EuMC '13), pp. 1067-1070, IEEE, Nuremberg, Germany, October 2013.

[26] K. Agarwal, Y. X. Guo, Nasimuddin, and A. Alphones, "Dualband circularly polarized stacked microstrip antenna over RIS for GPS applications," in Proceedings of the IEEE International Wireless Symposium (IWS '13), pp. 1-4, IEEE, Beijing, China, April 2013.

[27] K. Agarwal, Nasimuddin, and A. Alphones, "Triple-band compact circularly polarised stacked microstrip antenna over reactive impedance meta-surface for GPS applications," IET Microwaves, Antennas \& Propagation, vol. 8, no. 13, pp. 10571065, 2014. 

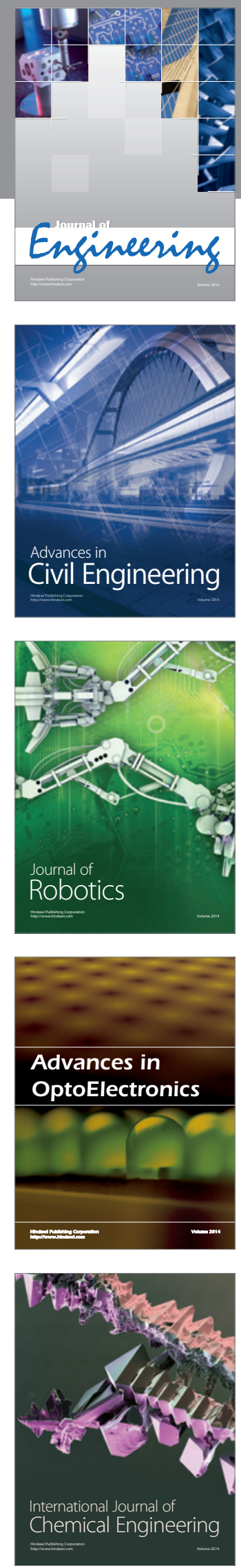

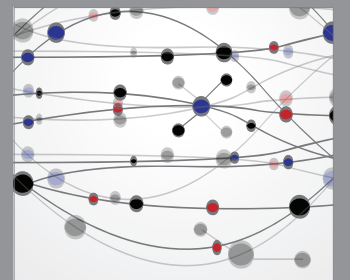

The Scientific World Journal
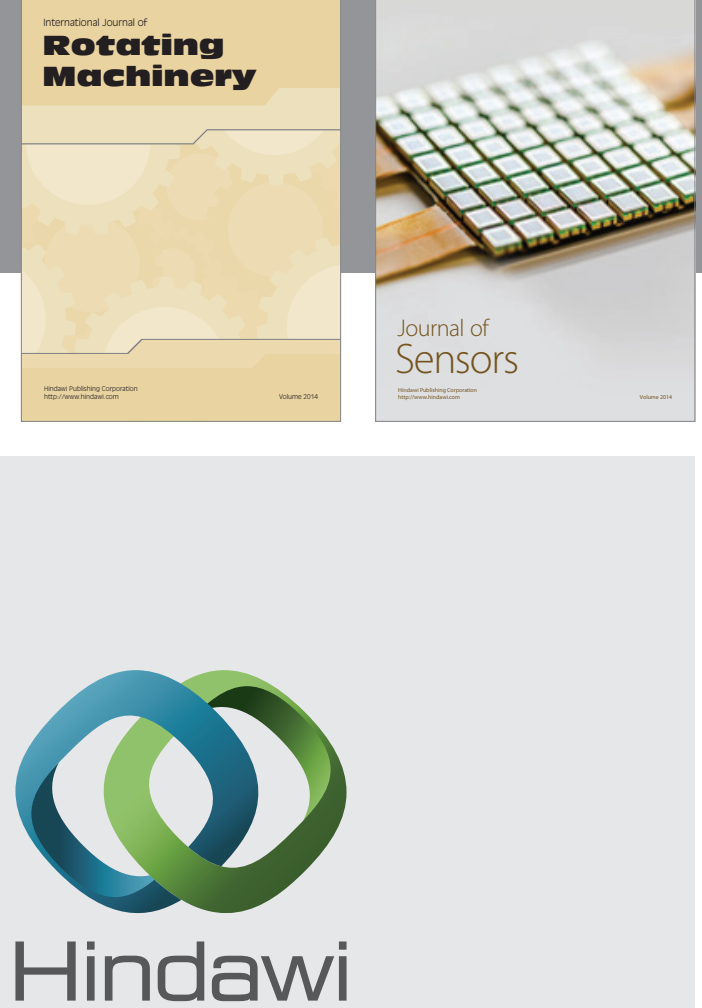

Submit your manuscripts at http://www.hindawi.com
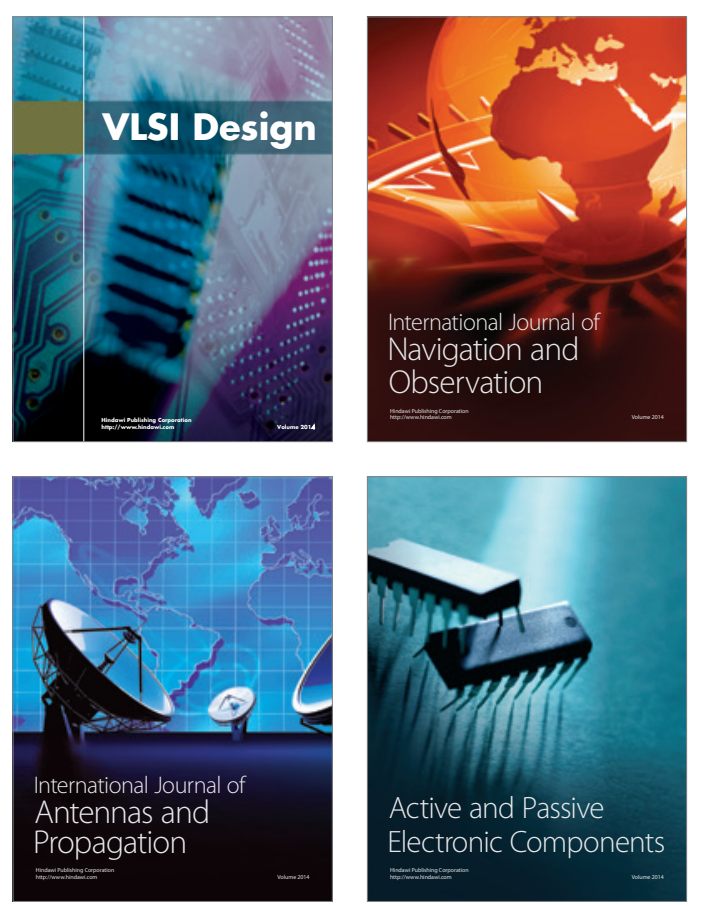
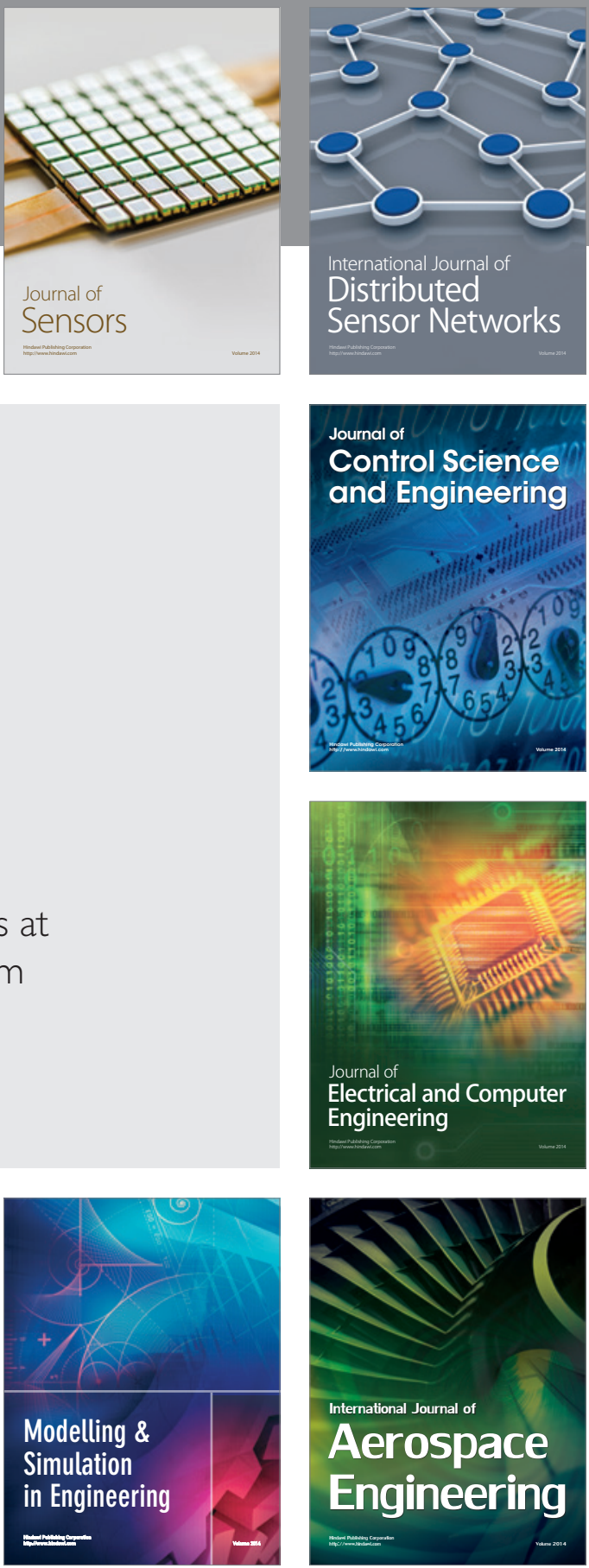

Journal of

Control Science

and Engineering
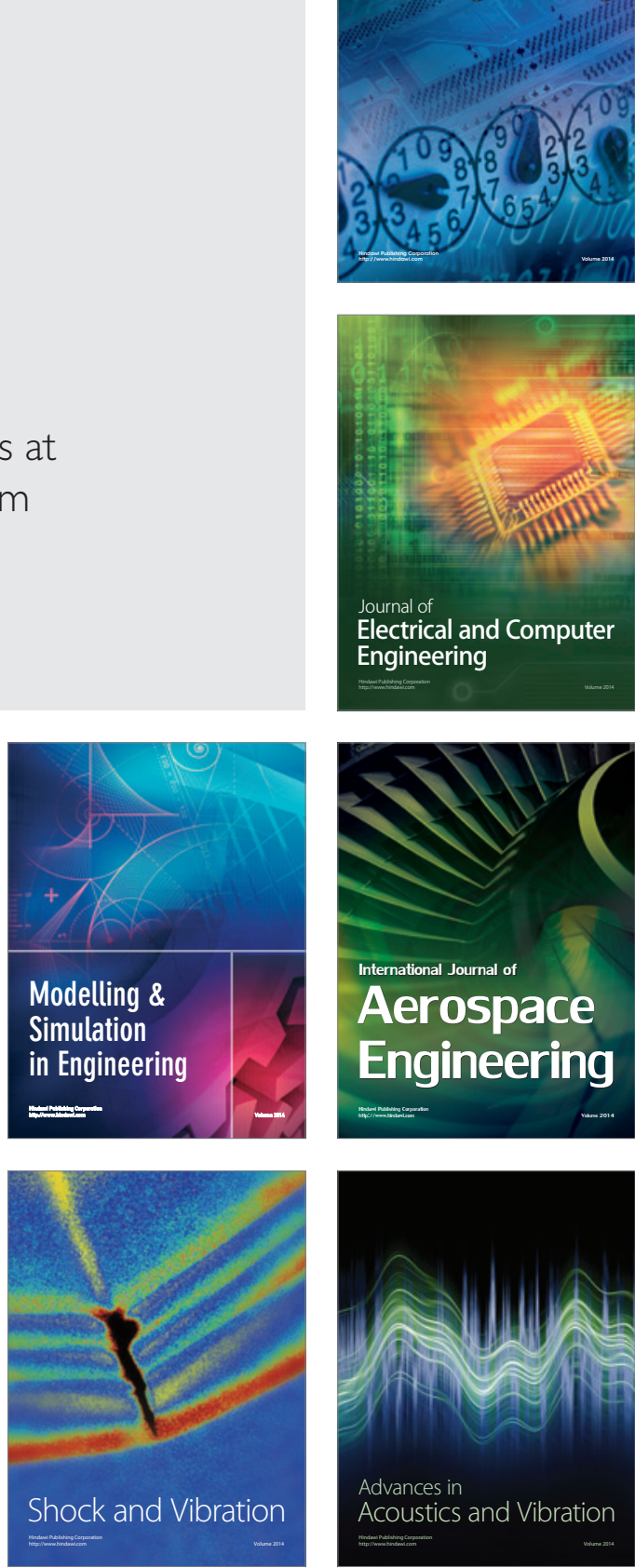